\title{
LA BENEFICENCIA ONUBENSE EN EL SELLO DURANTE LA GUERRA CIVIL ESPAÑOLA. PARTE II
}

\author{
José Eugenio Guerra González
}

Licenciado en Enfermería. Doctorando 1er. Año Enfermería y Cultura de los Cuidados. Hospital Juan Ramón Jiménez

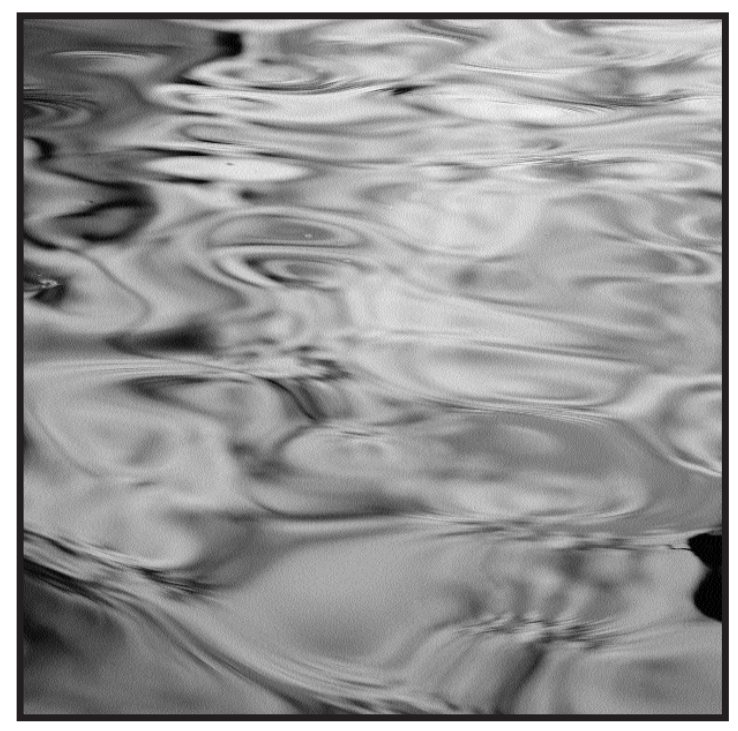

\section{CHARITY AND STAMPS IN HUELVA DURING THE SPANISH CIVIL WAR. PART II}

$\mathrm{T}$

This work continues the course of investigation started years ago with Postal History and stamps being considered a rich and interesting source for the study of the configuration of Nursing History. Given the fact that there are few studies at a national level, it is necessary to identify, describe and contextualise care and nursing phenomena in philatelic issues, in this case charity ones.

From the study of these local charity-aimed stamps issued during the Spanish Civil War, using qualitive research techniques -particularly document observation in Huelva local archives, we verified that 34 towns in Huelva issued charity stamps during this period, with 95 varieties (coats of arms, monuments, motherhood, etc.). Of the 17 towns studied in the current Part II, there is no documental proof of connection between the stamps issue and the use of money collected for charities, whilst in Part I connection was found for 5 towns (Ayamonte, Cartaya, Cortegana, Isla Cristina and Trigueros).

These "charity stamps" contributed to finance and manteinance of dining places and other charities, where poor people, women or children in need and prisoners were given food, clothing and sheltering.

Key words: stamps, philately, care, nursing, postal history and charity.

\section{RESUMEN}

Este trabajo continúa la línea de investigación iniciada hace años donde se considera a la Historia Postal, al sello como una amplia e interesante fuente de estudio para la construcción de la Historia de la Enfermería. Ante los escasos estudios a nivel nacional se hace necesario identificar, describir y contextualizar fenómenos cuidadores o enfermeros en las emisiones filatélicas, en este caso las denominadas "de beneficencia".

Del estudio de estos sellos de ámbito local y carácter benéfico emitidos durante la Guerra Civil Española y utilizando técnicas de investigación cualitativa, en especial la observación documental en Archivos Municipales onubenses se constata: 34 localidades onubenses emitieron sellos benéficos en este periodo, 95 variedades (escudos, monumentos, motivos maternales etc.). De las 17 localidades estudiadas en esta Parte II no existe constancia documental expresa de la relación entre la emisión de estos y el destino de su recaudación para el sufragio de distintas obras de beneficencia, no habiendo ocurrido esto en la Parte I, en donde en 5 localidades se encontró vínculos (Ayamonte, Cartaya, Cortegana, Isla Cristina y Trigueros).

Estos sellos "de beneficencia" contribuyeron al sufragio y mantenimiento entre otras obras de las denominadas cocinas económicas, comedores 
benéficos etc., donde se atendían a las gentes más necesitadas de toda sociedad en conflicto, mujeres, niños, ancianos y prisioneros en sus más básicas necesidades, alimentación, vestimenta y cobijo.

Palabras clave: sellos, filatelia, cuidados, enfermería, historia postal y beneficiencia.

\section{PRESENTACIÓN}

El presente trabajo, La Beneficencia Onubense en el sello durante la Guerra Civil Española, Parte II, es continuación de una línea de investigación comenzada hace varios años (Historia de la Enfermería: Reflejo en la Filatelia Mundial, 1.999), que presenta como objetivo primordial el dejar constancia de cómo la Historia Postal, la Filatelia y en definitiva el mundo del sello de correos en sus distintas manifestaciones (cartas, postales etc...), se erige en una original, valiosa y aun por explorar fuente de información para el estudio y construcción de nuestra propia Historia de Enfermería. En esta ocasión, es la Beneficencia Onubense durante la Guerra Civil Española el fenómeno que pretendo describir en la medida que los ejemplares filatélicos y la documentación existente en las distintas fuentes de información a las que he asistido al respecto lo han permitido.

\section{INTRODUCCIÓN}

El sello de correos presenta en primera instancia la utilidad de pagar de forma previa el franqueo y derechos de certificación de la correspondencia, siendo la Filatelia además de una afición por coleccionar sellos un "arte", arte de recorrer la Historia de la Humanidad, de las distintas culturas, pueblos y legados socioculturales. La primera emisión en el mundo de un sello fue en Gran Bretaña (1-51.840), en España tuvo lugar e1 1 de Enero de 1.850 , desde entonces más de 4.000 sellos han sido emitidos solo en nuestro país, ofreciendo una idea del volumen de ejemplares filatélicos que existen, ofreciendo el primer fenómeno enfermero en la emisión A favor de la Cruz Roja Española (16-91.926), cuyo motivo del sello o ilustración era la Reina Victoria Eugenia de Battemberg con uniforme de enfermera. La última referencia a nuestra profesión hace escasamente un año (8-5-2002), en el que se conmemoró el $175^{\circ}$ Aniversario del nacimiento del Dr. Federico Rubio y Galli, fundador de la primera Escuela de Enfermería, Sta. Isabel de Hungría, y en donde el motivo del sello o ilustración es su busto y el de dos enfermeras en plena actividad cuidadora.

Ante la necesidad de ir construyendo nuestra Historia de la Enfermería resulta imprescindible comenzar a explorar nuevas fuentes de investigación histórica, existiendo en la prolífera Historia Postal Española con más de 161 años de vigencia una emisiones denominadas "de beneficencia", de cuyo estudio histórico y filatélico surgirán a buen seguro relevantes aportaciones de lo que supusieron para la sociedad y el colectivo asistencial de la época. Escasos son los trabajos realizados en este sentido, destacando la labor de la Sr. Ma . Teresa Miralles Sangro (enfermera y docente en la Escuela de Enfermería de Alcalá de Henares, Madrid), cuyos trabajos han versado en la descripción de ejemplares filatélicos y su nexo con los cuidados, así como la difusión de la relación entre cuidados, enfermería y filatelia.

\section{OBJETIVOS}

- Mostrar como la Historia Postal, la Filatelia, el mundo del sello y las emisiones locales benéficas se erige en una amplia e interesante fuente de estudio para la construcción de la Historia de la Enfermería, respecto al fenómeno de la Beneficencia en Huelva durante la Guerra Civil Española.

- Identificar y describir filatélicamente los sellos locales y de carácter benéfico emitidos en Huelva y provincia durante la Guerra Civil Española.

- Identificar las aportaciones de estas emisiones al contexto socio-sanitario de la época en Huelva y provincia.

\section{METODOLOGÍA}

\section{Fuentes de Investigación}

La Historia Postal Filatélica, la Filatelia es la fuente de investigación utilizada, que según los criterios taxonómicos de clasificación de fuente de investigación histórica de Julio Aróstegui -posición, intención e información cualitativa y cuantitativa-, nos llevaría a contemplarla como fuente de 
de índole Cultural (no verbal, audiovisual capaz de trasmitir una información), Intencional, testimonial (al servir de franqueo a la correspondencia para fines recaudatorios), Seriada (susceptible de ser clasificados atendiendo a diversos criterios) y Directa (vinculada directamente con el objeto de estudio de la investigación, el sello de ámbito local y carácter benéfico).

\section{Técnicas de Investigación.}

Se han utilizado técnicas de índole cualitativa, primando la Observación Documental efectuada en 17 Archivos Municipales de localidades onubenses, el Archivo de la Diputación Provincial de Huelva, el Fondo Municipal Díaz Hierro, así como publicaciones y establecimientos especializados en Filatelia, prensa local de la época y la relevante producción surgida de entrevistas con gentes de edad que vivieron padeciendo in situ el conflicto bélico y sus consecuencias, amparándose en el fenómeno de la Beneficencia.

\section{RESULTADOS.}

En la Zona Republicana el origen de estos sellos benéficos está determinado por el Decreto de la Presidencia del Consejo de Ministros (23-121936), por el que se crean en las provincias españolas los Consejos provinciales, en cuyo articulado sobre "Competencias de los Consejos provinciales" aunque no se menciona expresamente que el Gobierno otorga capacidad a estos Consejos para emitir sellos, los mismos se amparan en esta omisión para hacerse con esta función, quedando reflejado en su articulado cuarto, punto E. A su vez, como tampoco se menciona explícitamente la capacidad de emitir sellos entre las funciones que el Gobierno no puede delegar entre sus Consejos provinciales -artículo ocho-, entienden que se está autorizando. Las emisiones benéficas en esta zona republicana adquieren el nombre de Pro-Infancia, Pro-Hospitales, Socorro Rojo etc.

En Zona Nacional, estos sellos aparecen en Andalucía, propiciado por la situación de escasez económica, la situación bélica y la labor del General Queipo de Llano con su política social en su vertiente asistencial y laboral (creación de comedores, reacio a implantar el racionamiento, iniciativa del Plato Único y Día sin Postre etc.), y como no a la emisión de sellos municipales obligatorios en la correspondencia de Sevilla de 5 cts., que mitigara la difícil y extrema situación por la que se encontraba la Hacienda Municipal en sus atenciones a la Beneficencia (19-8-1936, ptos. 4, 5). La denominación de las emisiones de carácter benéfico y local en este bando Nacional se denominaban Auxilio de Invierno, Frente y Hospitales, Cruzada contra el frío...

Las emisiones de estos sellos locales y benéficos se efectuaron en 51 provincias, en total 690 localidades españolas de las que se tiene constancia.

En Huelva fueron 34 localidades las que emitieron este tipo de sellos a falta de nuevos hallazgos, representando un total de 95 tipos de ejemplares filatélicos en base al motivo de emisión (escudos locales, monumentos, composiciones tipográficas, escenas familiares y cotidianas etc.). En este trabajo sólo se abordan 17 de estas localidades (Aracena, Bonares, Cala, Cerro de Andevalo, Cumbres Mayores, Encinasola, Escacena del Campo, Galaroza, Gibraleón, Hinojos, Jabugo, Paterna del Campo, Riotinto, Rosal de la Frontera, Sta. Ana la Real, Valverde del Camino y Villalba del Alcor), configurando un total de 49 sellos. En todas ellas no se ha encontrado expresamente vínculo alguno entre las recaudaciones hechas por la venta de estos ejemplares filatélicos y el empleo de las mismas en obras asistenciales, no así en las localidades onubenses estudiadas en la Parte I, donde en cinco de ellas (Ayamonte, Cortegana, Cartaya, Isla Cristina y Trigueros), ha sido posible encontrado constatación, referencia expresa a la relación entre estas emisiones filatélicas benéficas y el fin de la recaudación obtenida, aspecto este que paso a detallar:

1. AYAMONTE. El Ayuntamiento solicita al Gobierno Central (18-7-1937), autorización para la emisión de sellos de 5 cts. en la correspondencia para sufragar el abastecimiento de la Cocina Económica de la localidad, siendole concedido (98-1937).

2. CORTEGANA. El Ayuntamiento reconoce la labor y éxito de las Cocinas Económicas, pero también su imposibilidad para sufragar los gastos que genera, efectuando petición a instancias superiores (10-11-1936), para la implantación de unos sellos de entre 0.10 cts. y 1.5 ptas., de carácter 
obligatorio con el único objetivo de destinarlos a fines benéficos y humanitarios.

3. CARTAYA. El Estado de cuentas de las Cocinas Económicas de Cartaya (1937), ofrece un total de 21.500 raciones de comida servidas y por otro lado en los apartados Ingresos y Gastos en metálico y especies, ofrece por el concepto venta de sellos un total de 4.909 ptas., aproximadamente el $30 \%$ de la recaudación de estos establecimientos.

4. ISLA CRISTINA. La Junta del Sello hace entrega a la Junta de Auxilio Alimenticio a los Necesitados (14-9-1937), de un total de 5.200 sellos (5, 10, 50 cts. y 1 ptas.), por valor de 1.289 ptas. El Delegado Provincial de Auxilio Social en Huelva afirma (30-8-1937), que el sello Pro-Isla Cristina fue creado con el objeto de facilitar alimentos a todas aquellas personas que, por cualquier causa justificada se encontraran imposibilitadas para atender a su sustento y al de su familia por carecer de recursos a causa de paro forzoso, imposibilidad física etc.

5. TRIGUEROS. El Ayuntamiento comunica al Excmo. Sr. Gobernador General (28-10-1937), que por acuerdo se acepta la propuesta del Presidente de la Junta de Auxilio a los Necesitados de Huelva para establecer desde el $1^{\circ}$ de Enero un timbre de 5 cts. cuyos ingresos se destinarían al "Comedor Infantil" de la localidad. El 15 de Noviembre de 1.937 el Gobierno Civil de la Provincia de Huelva comunica al Alcalde de Trigueros que su solicitud ha sido denegada.

\section{CONCLUSIONES.}

La Historia Postal, la Filatelia, el mundo del sello y en particular estas emisiones filatélicas denominadas "de beneficencia" se comportan como una fuente más de estudio, aún por descubrir en su totalidad, para la construcción de la Historia de la Enfermería, de la Historia de los Cuidados, en este caso en el ámbito de la Beneficencia y en el periodo de la Guerra Civil Española, convirtiéndose la emisión de estos en uno de los ejes fundamentales de la política social y sanitaria de la España en plena Guerra Civil, con fines además del propagandístico a la creación, sufragio y mantenimiento de establecimientos benéficos y programas de índole asistencial.
Surgen ante el vacío normativo existente en cuánto a que de forma expresa no se menciona quienes tienen la obligación de emitir estos sellos (Gobierno, Consejos provinciales, etc.), por lo que estos últimos aprovechan la oportunidad para obtener más fondos para la reconstrucción de su localidad, demostrando la sensibilidad para con los más desfavorecidos de la sociedad.

El estudio pormenorizado de estas emisiones benéficas ofrece una panorámica bastante acertada de la situación social y asistencial que existía en la época en esas localidades onubenses (desastrosa, calamitosa), a quienes iban dirigidas estas medidas (niños, huérfanos, mujeres, ancianos, heridos, prisioneros y excombatientes), por quienes eran llevadas a cabo (mujeres jóvenes y de mediana edad sin relevancia social, ya fuera por iniciativa propia o en base a ideales y motivaciones políticas y religiosas), que se ofrecía (alimentación, cobijo y asistencia socio-sanitaria), en donde se realizaban (establecimientos benéficos, comedores, cocinas, asilos, casas de acogida, casas cuna etc.), ya fueran dirigidas por el propio Régimen o derivadas de congregaciones religiosas.

No debemos de olvidar al contemplar la correspondencia en donde se insertaban estos ejemplares filatélicos benéficos que aunque en su mayoría circulaban por territorio nacional, aquellos que se enviaban más allá de nuestras fronteras (ex-colonias, países extranjeros), se convertían en representantes y vehículos fidedignos de una realidad penosa que acontecía con todas sus consecuencias en nuestro país.

Es evidente que estamos ante una amplia, interesante y porqué no original fuente de estudio para la construcción de la Historia de los Cuidados, pero también hay que admitir que aún están por descubrir todas aquellas aportaciones que para el colectivo enfermero pueden emanar de su estudio, de ahí que se haga necesario el que surjan grupos de investigación que en esta línea vayan identificando las distintas vertientes a abordar. Somos pocos los profesionales atraídos en esta línea y trabajamos de forma individual y a un nivel básico, es decir contemplando tan solo la identificación y descripción somera de los ejemplares filatélicos que representaban fenómenos cuidadores y/o enfermeros ya fuera en la filatelia española o a 
nivel mundial. Un siguiente nivel sería la contextualización no ya de ejemplares filatélicos aislados, sino de emisiones como las que este estudio ofrece, en donde se exploren nexos entre estas emisiones y la repercusión tenida en la sociedad, en las gentes.

Desde este trabajo invito a todos los compañeros que estén interesados en trabajar en esta línea de investigación que considera a la Historia Postal, a la Filatelia como fuente de estudio para la construcción de la Historia de la Enfermería, la Historia de los Cuidados, se pongan en contacto con el que esto suscribe.

\section{BIBLIOGRAFÍA}

- ARÓSTEGUI, J. (1995) La investigación histórica: teoría y método. Crítica, Barcelona.

- Catálogo de los sellos "locales" emitidos durante la Guerra Civil Española (1936-39). Correos y Telégrafos. Edita FESOFI (Federación Española de Sociedades Filatélicas). 1995.

- COLÁS, M. Censures, marques e correspondances de la Guerra Civile d'Espagne.

- ESPINOSA MAESTRE, FCO. (1996) La Guerra Civil en Huelva. Diputación Provincial de Huelva.

- GALVEZ, M. (1960) Catálogo Especializado de España de Centenario.
- GÓMEZ GUILLAMON (1977) The Republican Local War Tax Stamps (1.936-39).

- GÓMEZ MORENO, J. (1992), Nerva. Historia de un pueblo. SAND, Artes Gráficas. Sevilla.

- GUERRA GONZÄLEZ, J.E. (2001), - La Beneficencia -. En: Historia de la Enfermería. Reflejo en la Filatelia Mundial. Actas del V Congreso Nacional de Historia de la Enfermería. Sevilla.

- Híades. Revista de Historia de la Enfermería, n. ${ }^{\circ} 7$. Septiembre - 2000 .

- MONTSENY, A. Los sellos benéficos y locales de España. 1959-63. Revista "Madrid Filatélica").

- RODRIGUEZ GUILLEN, A. (2001). La Guerra Civil en Aroche. Centro de Investigación Sancho IV.

- SHELLEY, R. The Postal Historie of the Spanish Civil War.

- SILES, J. (1999) Historia de la Enfermería. Aguaclara, Alicante.

- SUGRAÑES GÓMEZ, E.J., (1998), La Milagrosa y las Hijas de la Caridad en Huelva. Huelva.

\section{Localización y adquisición de ejemplares.}

- Filatelia Marina (Huelva).

- Filatelia San José (Huelva).

- Mercado del Coleccionismo "El Cabildo" (Sevilla).

- Estudio Filatélico "Mario Mirmán” (Sevilla).

- Filatelia Hernando Colón (Sevilla).

- Filatelia Costa Blanca (Alicante).

\section{EJEMPLARES FILATÉLICOS BENÉFICOS DE HUELVA Y PROVINCIA EMITIDOS DURANTE LA GUERRA CIVIL ESPAÑOLA.}

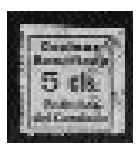

Bollullos del Condado.

Cocinas Benéficas.

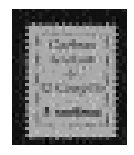

El Campillo. Cocinas benéficas.

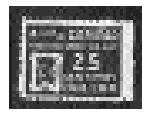

Huelva.

Asociación de Caridad.

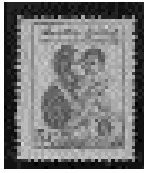

Bollullos del Condado.

Auxilio Social.

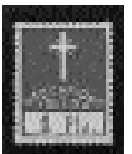

Cartaya.

Pro-Caridad.

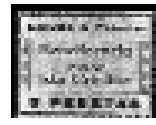

Isla Cristina.

Beneficencia 